\title{
A Prospective, Double Blind, Randomised, Placebo Controlled Trial Evaluating Acetyl-L-Carnitine (ALCAR) for the Prevention of Distal Symmetric Polyneuropathy in HIV Infected Individuals
}

Christian Herzmann ${ }^{1 *}$, Colette Smith ${ }^{4}$ Margaret A Johnson ${ }^{2}$, Patrick Byrne ${ }^{2}$, Giorgio Terenghi ${ }^{3}$, Yasotha Duraisamy ${ }^{3}$ and Mike Youle ${ }^{2}$

${ }^{1}$ Research Centre Borstel, Germany

${ }^{2}$ Royal Free Centre for HIV Medicine, Royal Free Hospital, London, UK

${ }^{3}$ Blond McIndoe Laboratories, Tissue Injury \& Repair Research Group, University of Manchester, UK

${ }^{4}$ Research Department of Infection and Population Health, UCL, London, UK

\begin{abstract}
Background: Nucleoside reverse transcriptase inhibitors (NRTIs) are currently an essential part of highly active antiretroviral therapy (HAART) for the treatment of HIV. However, the use of some dideoxynucleotide analogues may be limited by mitochondrial toxicity leading to distal symmetric polyneuropathy (DSP). Acetyl-L-Carnitine (ALCAR) has been investigated for the treatment of existing DSP but the potential for ALCAR to prevent DSP is unknown.

Methods: In this double blind, placebo controlled trial 43 HIV infected, antiretroviral naïve individuals were randomised to receive either ALCAR or placebo in addition to stavudine (as stavudine-XR, a sustained release formulation), tenofovir and efavirenz for 48 weeks. Development of DSP was assessed clinically and histologically by Protein Gene Product (PGP) staining of the epidermis. Quality of life (QOL) was measured during the study with the MOS-HIV Health Survey and the QUROQOL Score questionnaires.
\end{abstract}

Results: Twenty one subjects in each treatment arm were followed through 48 weeks. Discontinuation rates for stavudine and ALCAR versus placebo were similar in both groups. No differences were found for histological examination or clinical assessment of DSP; whilst the safety profile of ALCAR was comparable to placebo.

Conclusions: At 48 weeks the prophylactic administration of ALCAR with HAART to prevent DSP was no different than placebo, with a similar safety profile.

Keywords: HIV; Polyneuropathy; NRTI; Mitochondrial toxicity; Protein Gene Product; Immunostaining; Acetyl-L-Carnitine; EUROQOL; MOS-HIV

\section{Introduction}

Current highly active antiretroviral therapy (HAART) for the treatment of HIV infection is associated with long term side effects. Up to one third of patients treated with nucleoside reverse transcriptase inhibitors (NRTIs) experience peripheral neuropathic side effects [1]. This distal symmetric polyneuropathy (DSP) is mainly caused by some dideoxynucleoside analogues such as didanosine and stavudine. It appears that these compounds interfere with intracellular oxidative metabolism by reducing DNA synthesis within neuronal mitochondria [2-6] resulting in die-back of the peripheral nerve axons which leads to the affected areas showing reduced cutaneous innervation on histology $[7,8]$.

Painful DSP frequently leads to discontinuation of NRTIs [1]. Symptomatic treatment with tricyclic antidepressants, anticonvulsants and other drugs has been tried in HIV infected subjects with distal symmetric polyneuropathy (DSP) with limited success [9]. Unfortunately, no treatment options are yet licensed to treat or prevent the underlying neuronal damage.

In previous trials patients treated with Acetyl-L-Carnitine (ALCAR) reported a symptomatic improvement of existing DSP [10-13]. One study also found significant histological improvement in cutaneous innervation within the dermis, epidermis and sweat glands [10].

ALCAR is an ester of L-Carnitine that is generated from Carnitine and acetyl-CoA through a specific Carnitine acetyl transferase. This enzyme is mainly found in mitochondria of nervous tissue, heart, brown fat and spermatozoa. ALCAR is a transport molecule for free fatty acids and an important acetyl-group donor in high-energy metabolism and free fatty acid beta-oxidation $[12,14]$. It has analgesic properties and has been shown to improve pain scores in a placebocontrolled trial in diabetic subjects with peripheral polyneuropathy $[15,16]$. Previous investigations have shown an effect on neuropathy related glial cell line-derived neurotrophic factors and Artemin, an enhancing effect on Nerve Growth Factor (NGF) and peripheral nerve regeneration [17-20].

Apart from its mitochondria related properties the intravenous application of ALCAR has been found to increase the CD4 lymphocyte counts in subjects co-infected with HIV and hepatitis $\mathrm{C}$ who were not on standard HIV therapy [21]. This effect may have been due to a reduced frequency of apoptosis of CD4 and CD8 cells associated with ALCAR administration $[2,6,21]$. ALCAR has also been suggested

*Corresponding author: Christian Herzmann, Research Centre Borstel, Parkallee 35, 23845 Borstel, Germany, Tel: +49-4537-188-677; Fax +49-4537-188-713; E-mail: cherzmann@fz-borstel.de

Received November 12, 2010; Accepted December 05, 2010; Published December 07, 2010

Citation: Herzmann C, Smith C, Johnson MA, Byrne P, Terenghi G, et al. (2010) A Prospective, Double Blind, Randomised, Placebo Controlled Trial Evaluating Acetyl-L-Carnitine (ALCAR) for the Prevention of Distal Symmetric Polyneuropathy in HIV Infected Individuals. J AIDS Clinic Res 1:108. doi:10.4172/2155-6113.1000108

Copyright: $\odot 2010$ Mentzer A, et al. This is an open-access article distributed unde the terms of the Creative Commons Attribution License, which permits unrestricted use, distribution, and reproduction in any medium, provided the original author and source are credited. 
Citation: Herzmann C, Smith C, Johnson MA, Byrne P, Terenghi G, et al. (2010) A Prospective, Double Blind, Randomised, Placebo Controlled Trial Evaluating Acetyl-L-Carnitine (ALCAR) for the Prevention of Distal Symmetric Polyneuropathy in HIV Infected Individuals. J AIDS Clinic Res 1:108. doi:10.4172/2155-6113.1000108

Page 2 of 4

as a potential therapy for HIV-related lipodystrophy and metabolic syndrome [22-24].

In this study we investigated if the co-administration of ALCAR could prevent the development of DSP in HIV infected individuals commencing a HAART regimen with the potential to cause DSP.

\section{Methods}

The study was approved by the Royal Free Ethics Committee, London. Preliminary data indicated a change in percentage immunostaining on the epidermis in PGP from baseline to 48 weeks of $0.15 \%$ (standard deviation $0.15 \%$ ). Assuming to detect a worthwhile difference of $0.15 \%$ and assuming $90 \%$ power ( $5 \%$ significance level) resulted in a sample size of 20 subjects per treatment arm before allowing for loss to follow-up. All tests were two-sided. Randomisation was performed locally.

Between November 2003 and June 2005 HIV-1 infected, antiretroviral naïve adults commencing HAART at the Royal Free Hospital, London, UK were recruited for this double blind, randomised, placebo controlled trial. Subjects were excluded if there were signs of pre-existing peripheral polyneuropathy (diminished ankle reflexes or reduced sense of vibration), a history or evidence of potentially neurotoxic conditions (e.g. alcohol dependency, use of isoniazid or vincristine, diabetes mellitus, vitamin B12 deficiency) or administration of potential treatments for neuropathy two weeks prior to enrolment (e.g. tricyclic antidepressants, anticonvulsants, steroids, immune modulators). Patients who had AIDS, had ever taken ALCAR before or L-carnitine within the last 6 months were excluded. A negative pregnancy test and adequate oral contraception were mandatory for women.

The study drug was ALCAR $3 \mathrm{~g}$ dosed once daily as six $500 \mathrm{mg}$ tablets or matching placebos.

The antiretroviral therapy was a standardized once daily regimen of tenofovir $300 \mathrm{mg}$, efavirenz $600 \mathrm{mg}$ and stavudine-XR. This prolonged release capsule (PRC) of stavudine was dosed as $100 \mathrm{mg}$ and $75 \mathrm{mg}$ for subjects above and below $60 \mathrm{~kg}$ body weight, respectively [25].

Elliptical one centimetre skin biopsies were taken from a standardized level one third the distance from the ankle to the knee, as previously described, at day 1 , week 24 and week $48[7,10]$. The tissue was fixed in Zamboni's solution for 6 hours at room temperature, then transferred to phosphate buffered saline $(\mathrm{pH} 7.2)$ and stored at $4^{\circ} \mathrm{C}$. The samples were processed at a central laboratory (Blond MacIndoe Research Laboratories, University of Manchester, UK). After freezing, $10 \mu \mathrm{m}$ slices were cut on a cryostat and dried over night at room temperature. Immunostaining using antisera for various neuronal markers were performed as follows: pan neuronal markers of nerve terminals (PGP), markers of small un-myelinated fibres (peripherin, IB4 lectin), markers of A delta and C fibres (CGRP, Substance P), markers of post-ganglionic sympathetic nerve fibres (VIP, TH, NPY), markers of Schwann cells (S100, GFAP) and markers of growth factors (NGF, NT-3). The fractional area of immunostained structures from a microscopic visual field was measured using a semi-automatic established system described before [10]. For each subject at each time point, 6 visual fields were evaluated and the mean percentage of immunostaining was calculated.

The primary study endpoint was change from baseline in total area of protein gene product (PGP) immunostaining of the epidermis at 48 weeks. Secondary endpoints included the proportion of patients requiring new analgesic agents, an assessment of the safety and tolerance at 24 weeks, change in HIV-1 plasma viral load and in immunological markers (CD4 and CD8 lymphocyte cell counts), neurological signs and symptoms and global pain assessment using questionnaires (MOS 30 and EuroQoL).

Both an intention-to-treat/exposed (ITT/e) analysis and an on-treatment (OT) analysis were performed, the latter including only patients that were still on the study drug at this time. As the outcome was numerical, a missing=failure approach was taken to account for missing samples. However, the lost to follow-up rates were low. Subjects who did not have a skin biopsy taken at week 48 were excluded from the analysis of immunostaining (Figure 1). For the primary endpoint, a two sample t-test with equal or unequal variances as appropriate was used. Fisher's exact test, (or a chi-square test where expected frequencies were $>5$ ) were used to compare categorical secondary endpoints and a two sample t-test were used to compare numerical secondary endpoints.

\section{Results}

Forty seven subjects were screened but 4 withdrew their consent prior to randomisation (Figure 1). Twenty two were assigned to receive ALCAR, one of whom did not start the study medication. Eighteen of 21 were still on study medication at week 48 . In the placebo group, 19 of 21 subjects were still on study drug at week 48. The intention to treat analysis included 21 subjects in each group who took at least one dose of ALCAR/placebo.

Baseline parameters were similar in both groups (Table 1). The CD4 cell count was slightly higher in the treatment group.

During the 48 -week period, stavudine-XR was discontinued by 4 individuals $(19.1 \%)$ of subjects after a mean of 181 days (range, 31266) versus 5 individuals (23.8\%) after a mean of 149 days (range, 22-335) in the ALCAR and placebo arm, respectively. The numbers who discontinued tenofovir were 2 (9.5\%) and 3 (14.3\%); 8 (38.1\%) and $10(47.6 \%)$ discontinued efavirenz in the ALCAR and placebo group, respectively during the 48 -week study period. The study drug (i.e. either ALCAR or placebo) was discontinued in 3 (14.2\%) (ALCAR group) versus $2(9.5 \%)$ (placebo group) individuals within the 48-week interval.

The changes of the median fractional area of immunostaining

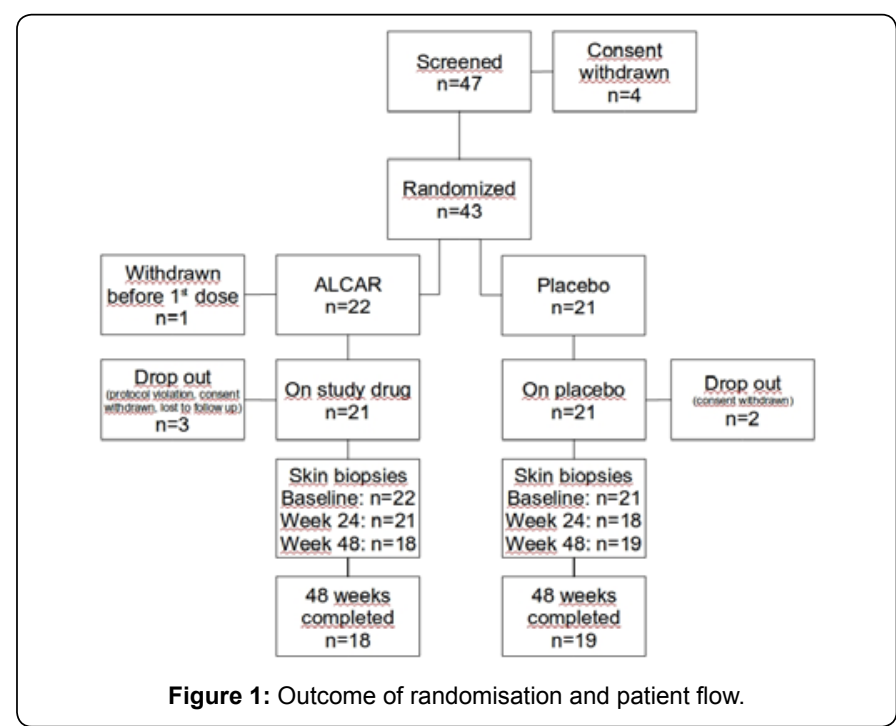


Citation: Herzmann C, Smith C, Johnson MA, Byrne P, Terenghi G, et al. (2010) A Prospective, Double Blind, Randomised, Placebo Controlled Trial Evaluating Acetyl-L-Carnitine (ALCAR) for the Prevention of Distal Symmetric Polyneuropathy in HIV Infected Individuals. J AIDS Clinic Res 1:108. doi:10.4172/2155-6113.1000108

Page 3 of 4

\begin{tabular}{|l|l|l|}
\hline Group & Placebo & ALCAR \\
\hline Number of subjects & 21 & 21 \\
\hline Male subjects $(\%)$ & $18(85.7)$ & $20(95.2)$ \\
\hline Median age $(\mathrm{Cl})$ & $39(31 ; 46)$ & $36(32 ; 41)$ \\
\hline Caucasian / African / Other & $16 / 2 / 3$ & $17 / 2 / 2$ \\
\hline Mean BMI [kg / m²] (Cl) & $23.8(21.9 ; 25.6)$ & $22.9(21.3 ; 24.1)$ \\
\hline CDC category A / B / C & $10 / 9 / 2$ & $10 / 8 / 3$ \\
\hline $\begin{array}{l}\text { Median HIV viral load [copies } \\
\text { / ml] (Cl) }\end{array}$ & $100001(31100 ;$ & $111000(80200 ; 325000)$ \\
\hline $\begin{array}{l}\text { Median CD4 cell count [cells/ } \\
\text { Hl] (Cl) }\end{array}$ & $179000)$ & $218(74 ; 272)$ \\
\hline
\end{tabular}

$\mathrm{Cl}$ : Confidence interval

Table 1: Baseline characteristics.

\begin{tabular}{|l|l|l|l|l|}
\hline Marker & Week & ALCAR group & Placebo group & P value \\
\hline \multirow{2}{*}{ PGP } & 24 & $5.6(2.6 ; 8.2) n=20$ & $3.3(2.4 ; 6.4) n=17$ & 0.23 \\
\cline { 2 - 6 } & 48 & $3.7(1.2 ; 6.7) n=18$ & $4.1(1.9 ; 9.5) n=19$ & 0.63 \\
\hline \multirow{2}{*}{ CGRP } & 24 & $0.0(0 ; 1.5) n=20$ & $1.0(0 ; 1.4) n=17$ & 0.66 \\
\cline { 2 - 6 } & 48 & $0.3(0 ; 1.5) n=18$ & $1.1(0 ; 3.0) n=19$ & 0.16 \\
\hline \multirow{2}{*}{ VIP } & 24 & $1.1(0 ; 1.9) n=7$ & $1.4(0 ; 2.2) n=7$ & 0.56 \\
\cline { 2 - 6 } & 48 & $0.6(0 ; 3.0) n=9$ & $3.4(1.7 ; 4.5) n=8$ & 0.14 \\
\hline
\end{tabular}

Table 2: Percentages of immunostaining for the main neuronal markers [median for each group; interquartile range IQR]. All percentages are multiplied by 1000 .

\begin{tabular}{|l|l|l|}
\hline Parameter & ALCAR group & Placebo group \\
\hline $\begin{array}{l}\text { Number }(\%) \text { of subjects with HIV-1 viral load } \\
<50 \mathrm{cc} / \mathrm{ml}\end{array}$ & $17(81)$ & $18(86)$ \\
\hline CD4 cell count (median increase per $\mu \mathrm{l}, \mathrm{IQR})$, & $119(67 ; 147)$ & $161(82 ; 256)$ \\
\hline $\begin{array}{l}\text { MOS-HIV physical health score (median } \\
\text { change, IQR) }\end{array}$ & $0(-2,1 ; 4.0)$ & $0(-0.9 ; 0.8)$ \\
\hline MOS-HIV mental score (median change, IQR) & $0.7(0 ; 0.51)$ & $0(-2.1 ; 5.1)$ \\
\hline $\begin{array}{l}\text { EUROQOL visual analogue scale (median } \\
\text { change, IQR) }\end{array}$ & $0(-1 ; 15)$ & $0(0 ; 13)$ \\
\hline EUROQOL score (median change, IQR) & $0(0 ; 0)$ & $0(0 ; 0)$ \\
\hline Number of subjects with lipodystrophy $(\%)$ & $4(22)$ & $3(16)$ \\
\hline
\end{tabular}

Table 3: Secondary endpoints at week 48. No endpoint reached statistical significance.

for PGP, CGRP and VIP did not reach statistical significance (Table 2). Measurement for the other applied antisera also did not show significant differences. The on-treatment analysis resulted in similar outcomes as the intention-to-treat / exposed analysis.

There was no difference in any secondary endpoint between the ALCAR and placebo group at week 48 (Table 3). The percentage of patients achieving HIV-1 viral loads $<50 / \mathrm{ml}$ and the changes in CD4 cell counts were similar in both groups. The quality of life as assessed by questionnaires (MOS-HIV physical health score, MOS-HIV mental health score, EUROQOL visual analogue scale, EUROQOL score) did not differ. ALCAR had no impact on the incidence of lipodystrophy.

No patient developed clinically detectable peripheral neuropathy. There were four serious adverse events in the placebo arm and five in the ALCAR arm. All events were considered not to be related to the study drug. In the ALCAR group the events were: possible abacavir hypersensitivity reaction, rectal mass associated with a rectal ulcer, community acquired pneumonia, medulla oblongata infarct, chest infection. In the placebo arm the events were: nodular malignant melanoma, perianal abscess, rash due to efavirenz, suicide attempt.

\section{Discussion}

Acetyl-L-Carnitine has been shown to be an effective pathogenesis based therapy for the antiretroviral toxic neuropathy $[10,11,26]$. Thus, attempting to prevent distal symmetric polyneuropathy by adding ALCAR to an antiretroviral regime appears tempting. The strategy is similar to the combination of isoniazid with pyridoxine to prevent neurotoxicity.

A recent in-vitro trial assessing the prophylactic effect of ALCAR on fetal rat neurons exposed to stavudine or didanosine showed a preventive effect only against neurotoxicity induced by didanosine not by stavudine [27]. Similarly, this double blind, placebo controlled, randomised trial did not show any prophylactic effect of ALCAR in vivo. Given that no differences in immunostaining for several neuronal markers were seen in either group, the occurrence of clinically relevant DSP was unlikely. Accordingly, no case of clinical DSP was diagnosed in either group. This incidence is lower than the published numbers of $10-35 \%$ but should be interpreted cautiously due to the small sample size $[1,28,29]$. Therapy with the DSP causing agent stavudine was discontinued by about one fifth of all patients within the study period of one year (ALCAR group: 19\%, placebo group: $23,8 \%$ ) whilst subjects taking ALCAR remained slightly longer on the NRTI although the finding was not significant. Only one subject in the ALCAR group complained of paraesthesia in his toes but DSP could not be confirmed. It appears that study subjects were very alert regarding any neuropathic symptoms and were willing to discontinue any potential neurotoxic drug after less than 48 weeks of treatment.

An increase of stavudine associated polyneuropathy has been shown after 2 years; shorter exposure led to DSP in 13\%, longer exposure in $29 \%$ [30]. In a South African study which examined drug toxicity severe enough to result in substitution of the causal agent by an alternative antiretroviral, the stavudine substitution rate rose steadily to $20.8 \%$ over a period of three years; during the first two years $6.2 \%$ discontinued the drug due to DSP. Thereafter, this rate remained stable but the drug was discontinued for different reasons during the third year, e.g. lipodystrophy and lactic acidosis [31].

Neurotoxicity to NRTIs appears to cumulate over the first two years leading to the highest morbidity and discontinuation rates within this period of exposure. This potential bias in the incidence of DSP is a weakness of the trial since it is possible that the prophylactic effects of ALCAR would only be seen if the observation period was to exceed 48 weeks.

Discontinuation rates for ALCAR (or placebo) were nearly as high as the rates for stavudine despite the fact that neither ALCAR nor placebos have significant side effects. Notably, the quality of life as assessed with questionnaires did not show any changes from baseline at weeks $12,24,36$ or 48 . It can therefore be assumed that motivating patients to continue a prophylactic medication with no recognisable subjective benefit was more challenging than motivating patients who seek relief from an illness. The high discontinuation rates may have led to an underestimation of the prophylactic effect of ALCAR. In summary, the study was underpowered to discover a significant difference in DSP between the treatment arms.

One other NRTI side effect that has been associated with mitochondrial toxicity is lipodystrophy. The beneficial effect of ALCAR for this condition has been hypothesised years ago $[2,6]$. However, in contrast to others we were unable to confirm a clinical benefit of prophylactic ALCAR administration [22]. Physicians were more reluctant to diagnose the condition than patients themselves but the numbers were similar in both treatment arms. In addition the quality of life did not differ between the groups. Again, the study may have been underpowered to detect subtle differences.

Previous studies described the ability of ALCAR to prevent lymphocytic apoptosis [23]. A small Italian trial treated $11 \mathrm{HIV} /$ hepatitis $\mathrm{C}$ co-infected patients with ALCAR but not with a standard antiretroviral regime. Under these conditions a significant rise of CD4 cell counts was noted after 4 months [24]. In our study, we could not confirm either a virological or an immunological benefit of ALCAR over 48 weeks compared to placebo. However, it is possible that the immunologic effect of ALCAR is too weak to be discovered when 
Citation: Herzmann C, Smith C, Johnson MA, Byrne P, Terenghi G, et al. (2010) A Prospective, Double Blind, Randomised, Placebo Controlled Trial Evaluating Acetyl-L-Carnitine (ALCAR) for the Prevention of Distal Symmetric Polyneuropathy in HIV Infected Individuals. J AIDS Clinic Res 1:108. doi:10.4172/2155-6113.1000108

Page 4 of 4

administered with conventional HAART. Given our findings in this small cohort, ALCAR can not be recommended for the treatment of HIV itself or HIV associated immunodeficiency.

ALCAR remains an option for the treatment rather than the prevention of antiretroviral toxic neuropathy for some patients. However, the current data do not support a prophylactic administration in combination with HAART.

\section{Acknowledgements}

We thank the patients and staff of the lan Charleson Day Centre at the Royal Free Hospital, London, for their active support of the trial and the Copenhagen HIV Programme, Denmark, for its collaboration.

\section{Conflict of Interest}

Mike Youle has received conference travel expenses from Sigma-Tau. The other authors declare no conflicting interests.

\section{References}

1. Moore RD, Wong WM, Keruly JC, McArthur JC (2000) Incidence of neuropathy in HIV-infected patients on monotherapy versus those on combination therapy with didanosine, stavudine and hydroxyurea. AIDS 14: 273-278.

2. Brinkman K, ter Hofstede HJ, Burger DM, Smeitink JA, Koopmans PP (1998) Adverse effects of reverse transcriptase inhibitors: mitochondrial toxicity as common pathway. AIDS 12: 1735-1744.

3. Chen $\mathrm{CH}$, Vazquez-Padua M, Cheng YC (1991) Effect of anti-human immunodeficiency virus nucleoside analogs on mitochondrial DNA and its implication for delayed toxicity. Mol Pharmacol 39: 625-628.

4. Keilbaugh SA, Prusoff WH, Simpson MV (1991) The PC12 cell as a model for studies of the mechanism of induction of peripheral neuropathy by anti-HIV-1 dideoxynucleoside analogs. Biochem Pharmacol 42: R5-8.

5. Lewis W, Dalakas MC (1995) Mitochondrial toxicity of antiviral drugs. Nat Med 1: 417-422.

6. Brinkman K, ter Hofstede J (1999) Mitochondrial toxicity of nucleoside analogue reverse transcriptase inhibitors: Lactic acidosis, risk factors and therapeutic options. AIDS Reviews 1: 140-147.

7. McCarthy BG, Hsieh ST, Stocks A, Hauer P, Macko C, et al. (1995) Cutaneous innervation in sensory neuropathies: evaluation by skin biopsy. Neurology 45 : 1848-1855.

8. Polydefkis M, Yiannoutsos CT, Cohen BA, Hollander H, Schifitto G, et al. (2002) Reduced intraepidermal nerve fiber density in HIV-associated sensory neuropathy. Neurology 58: 115-119.

9. Kieburtz KD, Seidlin M, Lambert JS, Dolin R, Reichman R, et al. (1992) Extended follow-up of peripheral neuropathy in patients with AIDS and AIDSrelated complex treated with dideoxyinosine. J Acquir Immune Defic Syndr 5: 60-64.

10. Hart AM, Wilson AD, Montovani C, Smith C, Johnson M, et al. (2004 ) AcetylI-carnitine: a pathogenesis based treatment for HIV-associated antiretroviral toxic neuropathy. AIDS 18: 1549-1560

11. Herzmann C, Johnson MA, Youle M (2005) Long-term effect of acetyl-Lcarnitine for antiretroviral toxic neuropathy. HIV Clin Trials 6: 344-350.

12. Bremer J (1990) The role of carnitine in intracellular metabolism. J Clin Chem Clin Biochem 28: 297-301.

13. Youle M, Osio M, (2007) A double-blind, parallel-group, placebo-controlled, multicentre study of acetyl I-carnitine in the symptomatic treatment of antiretroviral toxic neuropathy in patients with HIV-1 infection. HIV Medicine 8: 241-250.

14. Colucci W, Gandour R (1988) Carnitine acyltransferase: a review of its biology enzymology and bioorganic chemistry. Bioorg Chem 16: 307-334.

15. De Grandis D, Minardi C (2002) Acetyl-L-carnitine (levacecarnine) in the treatment of diabetic neuropathy. A long-term, randomised, double-blind, placebo-controlled study. Drugs R D 3: 223-231.

16. Onofrj M, Fulgente T, Melchionda D, Marchionni A, Tomasello F, et al. (1995) L-acetylcarnitine as a new therapeutic approach for peripheral neuropathies with pain. Int J Clin Pharmacol Res 15: 9-15.

17. Angelucci L, Ramacci MT, Taglialatela G, Hulsebosch C, Morgan B, et al (1988) Nerve growth factor binding in aged rat central nervous system: effect of acetyl-L-carnitine. J Neurosci Res 20: 491-496.

18. Fernandez E, Pallini R, Gangitano C, Del Fa A, Sangiacomo CO, et al. (1989) Effects of L-carnitine, L-acetylcarnitine and gangliosides on the regeneration of the transected sciatic nerve in rats. Neurol Res 11: 57-62.

19. McKay Hart A, Wiberg M, Terenghi G (2002) Pharmacological enhancement of peripheral nerve regeneration in the rat by systemic acetyl-L-carnitine treatment. Neurosci Lett 334: 181-185.

20. Vivoli E, Di Cesare Mannelli L, Salvicchi A, Bartolini A, Koverech A, et al. (2010) Acetyl-L-carnitine increases artemin level and prevents neurotrophic facto alterations during neuropathy. Neuroscience 167: 1168-1174.

21. Fouty B, Frerman F, Reves R (1998) Riboflavin to treat nucleoside analogueinduced lactic acidosis. Lancet. 352: 291-292.

22. Benedini S, Perseghin G, Terruzzi I, Scifo P, Invernizzi PL, et al. (2009) Effect of L-acetylcarnitine on body composition in HIV-related lipodystrophy. Horm Metab Res 41: 840-845.

23. Cifone MG, Alesse E, Di Marzio L, Ruggeri B, Zazzeroni F, et al. (1997) Effect of $\mathrm{L}$-carnitine treatment in vivo on apoptosis and ceramide generation in peripheral blood lymphocytes from AIDS patients. Proc Assoc Am Physicians 109: 146-153.

24. Moretti S, Alesse E, Di Marzio L, Zazzeroni F, Ruggeri B, et al. (1998) Effect of $L$-carnitine on human immunodeficiency virus-1 infection-associated apoptosis: a pilot study. Blood 91: 3817-3824.

25. FDA FaDA (2005) Label information for Zerit XR.

26. Hart AM, Terenghi G, Johnson MA, Youle M (2000) L-Acetyl-Carnitine (LAC) therapy increases cutaneous innervation and improves symptoms in antiretroviral therapy related HIV peripheral neuropathy. 3rd Internationa Workshop on Salvage Therapy in HIV Infection: Chicago.

27. Cherry CL, Mobarok M, Wesselingh SL, Fain R, Weinstock S, et al (2010) Ubisol-Aqua: coenzyme Q10 prevents antiretroviral toxic neuropathy in an in vitro model. Curr HIV Res 8: 232-239.

28. Kieburtz K, Simpson D, Yiannoutsos C, Max MB, Hall CD, et al. (1998) A randomized trial of amitriptyline and mexiletine for painful neuropathy in HIV infection. AIDS Clinical Trial Group 242 Protocol Team. Neurology 51: 16821688.

29. Moyle GJ, Sadler M (1998) Peripheral neuropathy with nucleoside antiretrovirals: risk factors, incidence and management. Drug Saf 19: 481-494

30. Scarsella A, Coodley G, Shalit P, Anderson R, Fisher RL, et al (2002) Stavudine-associated peripheral neuropathy in zidovudine-naive patients: effect of stavudine exposure and antiretroviral experience. Adv Ther 19: 1-8.

31. Boulle A, Orrel C, Kaplan R, Van Cutsem G, McNally M, et al (2007) Substitutions due to antiretroviral toxicity or contraindication in the first 3 years of antiretroviral therapy in a large South African cohort. Antivir Ther 12: 753-760. 